\title{
Statistical Analysis of Parameter Estimation for 2-D Harmonics in Multiplicative and Additive Noise
}

Huiming Peng, Jiawen Bian, Diwei Yang, Zhihui Liu, Hongwei Li*

School of Mathematics and Physics, China University of Geosciences

Wuhan 430074, China (*Email address: hwli@cug.edu.cn)

\section{Supplementary Data}

(A) The expression of $Q(\boldsymbol{\theta})$ in the proof of Theorem 2 .

$$
\begin{aligned}
Q(\boldsymbol{\theta})= & \sum_{m=1}^{M} \sum_{n=1}^{N}\left\{\sum_{k=1}^{p}\left[\mu_{s k}^{0} \cos \left(w_{m n}\left(\boldsymbol{\theta}_{k}^{0}\right)\right)-\mu_{s k} \cos \left(w_{m n}\left(\boldsymbol{\theta}_{k}\right)\right)\right]\right\}^{2} \\
& +\sum_{m=1}^{M} \sum_{n=1}^{N}\left\{\sum_{k=1}^{p}\left[\mu_{s k}^{0} \sin \left(w_{m n}\left(\boldsymbol{\theta}_{k}^{0}\right)\right)-\mu_{s k} \sin \left(w_{m n}\left(\boldsymbol{\theta}_{k}\right)\right)\right]\right\}^{2} \\
& +2 \sum_{m=1}^{M} \sum_{n=1}^{N} \sum_{k=1}^{p} x_{r}(m, n)\left[\mu_{s k}^{0} \cos \left(w_{m n}\left(\boldsymbol{\theta}_{k}^{0}\right)\right)-\mu_{s k} \cos \left(w_{m n}\left(\boldsymbol{\theta}_{k}\right)\right)\right] \\
& +2 \sum_{m=1}^{M} \sum_{n=1}^{N} \sum_{k=1}^{p} x_{c}(m, n)\left[\mu_{s k}^{0} \sin \left(w_{m n}\left(\boldsymbol{\theta}_{k}^{0}\right)\right)-\mu_{s k} \sin \left(w_{m n}\left(\boldsymbol{\theta}_{k}\right)\right)\right] \\
& +\sum_{m=1}^{M} \sum_{n=1}^{N}|x(m, n)|^{2}
\end{aligned}
$$

where

$$
\begin{aligned}
x(m, n) & =e(m, n)+\sum_{k=1}^{p}\left(s_{k}(m, n)-\mu_{s k}^{0}\right) e^{i w_{m n}\left(\boldsymbol{\theta}_{k}^{0}\right)} \\
w_{m n}\left(\boldsymbol{\theta}_{k}\right) & =m \lambda_{k}+n \mu_{k}+\phi_{k}
\end{aligned}
$$

(B) The expressions of the elements of $Q^{\prime}\left(\boldsymbol{\theta}^{0}\right)$ in the proof of Theorem 2.

$$
\begin{gathered}
Q^{\prime}\left(\boldsymbol{\theta}^{0}\right)=\left(Q_{k}^{\prime}\left(\boldsymbol{\theta}^{0}\right)\right)_{k=1,2, \ldots, p}=\left(Q_{1}^{\prime}\left(\boldsymbol{\theta}^{0}\right), Q_{2}^{\prime}\left(\boldsymbol{\theta}^{0}\right), \cdots, Q_{p}^{\prime}\left(\boldsymbol{\theta}^{0}\right)\right) \\
Q_{k}^{\prime}\left(\boldsymbol{\theta}^{0}\right)=\frac{\partial Q\left(\boldsymbol{\theta}^{0}\right)}{\partial \boldsymbol{\theta}_{k}}=\left(\frac{\partial Q\left(\boldsymbol{\theta}^{0}\right)}{\partial \mu_{s k}}, \frac{\partial Q\left(\boldsymbol{\theta}^{0}\right)}{\partial \phi_{k}}, \frac{\partial Q\left(\boldsymbol{\theta}^{0}\right)}{\partial \lambda_{k}}, \frac{\partial Q\left(\boldsymbol{\theta}^{0}\right)}{\partial \mu_{k}}\right) \\
\frac{\partial Q\left(\boldsymbol{\theta}^{0}\right)}{\partial \mu_{s k}}=2 \sum_{m=1}^{M} \sum_{n=1}^{N}\left[-x_{r}(m, n) \cos \left(w_{m n}\left(\boldsymbol{\theta}_{k}^{0}\right)\right)-x_{c}(m, n) \sin \left(w_{m n}\left(\boldsymbol{\theta}_{k}^{0}\right)\right)\right] \\
\frac{\partial Q\left(\boldsymbol{\theta}^{0}\right)}{\partial \phi_{k}}=2 \sum_{m=1}^{M} \sum_{n=1}^{N} \mu_{s k}^{0}\left[x_{r}(m, n) \sin \left(w_{m n}\left(\boldsymbol{\theta}_{k}^{0}\right)\right)-x_{c}(m, n) \cos \left(w_{m n}\left(\boldsymbol{\theta}_{k}^{0}\right)\right)\right] \\
\frac{\partial Q\left(\boldsymbol{\theta}^{0}\right)}{\partial \lambda_{k}}=2 \sum_{m=1}^{M} \sum_{n=1}^{N} m \mu_{s k}^{0}\left[x_{r}(m, n) \sin \left(w_{m n}\left(\boldsymbol{\theta}_{k}^{0}\right)\right)-x_{c}(m, n) \cos \left(w_{m n}\left(\boldsymbol{\theta}_{k}^{0}\right)\right)\right] \\
\frac{\partial Q\left(\boldsymbol{\theta}^{0}\right)}{\partial \mu_{k}}=2 \sum_{m=1}^{M} \sum_{n=1}^{N} n \mu_{s k}^{0}\left[x_{r}(m, n) \sin \left(w_{m n}\left(\boldsymbol{\theta}_{k}^{0}\right)\right)-x_{c}(m, n) \cos \left(w_{m n}\left(\boldsymbol{\theta}_{k}^{0}\right)\right)\right]
\end{gathered}
$$


(C) The expressions of the elements of $Q^{\prime \prime}\left(\boldsymbol{\theta}^{0}\right)$ in the proof of Theorem 2 .

$$
\begin{gathered}
Q^{\prime \prime}\left(\boldsymbol{\theta}^{0}\right)=\left(Q_{k l}^{\prime \prime}\left(\boldsymbol{\theta}^{0}\right)\right)_{k, l=1,2, \ldots, p}=\left[\begin{array}{cccc}
Q_{11}^{\prime \prime}\left(\boldsymbol{\theta}^{0}\right) & Q_{12}^{\prime \prime}\left(\boldsymbol{\theta}^{0}\right) & \cdots & Q_{1 p}^{\prime \prime}\left(\boldsymbol{\theta}^{0}\right) \\
Q_{21}^{\prime \prime}\left(\boldsymbol{\theta}^{0}\right) & Q_{22}^{\prime \prime}\left(\boldsymbol{\theta}^{0}\right) & \cdots & Q_{2 p}^{\prime \prime}\left(\boldsymbol{\theta}^{0}\right) \\
\vdots & \vdots & \ddots & \vdots \\
Q_{p 1}^{\prime \prime}\left(\boldsymbol{\theta}^{0}\right) & Q_{p 2}^{\prime \prime}\left(\boldsymbol{\theta}^{0}\right) & \cdots & Q_{p p}^{\prime \prime}\left(\boldsymbol{\theta}^{0}\right)
\end{array}\right] \\
Q_{k l}^{\prime \prime}\left(\boldsymbol{\theta}^{0}\right)=\frac{\partial^{2} Q\left(\boldsymbol{\theta}^{0}\right)}{\partial \boldsymbol{\theta}_{k} \partial \boldsymbol{\theta}_{l}^{T}}=\left[\begin{array}{cccc}
\frac{\partial^{2} Q\left(\boldsymbol{\theta}^{0}\right)}{\partial \mu_{s k} \partial \mu_{s l}} & \frac{\partial^{2} Q\left(\boldsymbol{\theta}^{0}\right)}{\partial \mu_{s k} \partial \phi_{l}} & \frac{\partial^{2} Q\left(\boldsymbol{\theta}^{0}\right)}{\partial \mu_{s k} \partial \lambda_{l}} & \frac{\partial^{2} Q\left(\boldsymbol{\theta}^{0}\right)}{\partial \mu_{s k} \partial \mu_{l}} \\
\frac{\partial^{2} Q\left(\boldsymbol{\theta}^{0}\right)}{\partial \phi_{k} \partial \mu_{s l}} & \frac{\partial^{2} Q\left(\boldsymbol{\theta}^{0}\right)}{\partial \phi_{k} \partial \phi_{l}} & \frac{\partial^{2} Q\left(\boldsymbol{\theta}^{0}\right)}{\partial \phi_{k} \partial \lambda_{l}} & \frac{\partial^{2} Q\left(\boldsymbol{\theta}^{0}\right)}{\partial \phi_{k} \partial \mu_{l}} \\
\frac{\partial^{2} Q\left(\boldsymbol{\theta}^{0}\right)}{\partial \lambda_{k} \partial \mu_{s l}} & \frac{\partial^{2} Q\left(\boldsymbol{\theta}^{0}\right)}{\partial \lambda_{k} \partial \phi_{l}} & \frac{\partial^{2} Q\left(\boldsymbol{\theta}^{0}\right)}{\partial \lambda_{k} \partial \lambda_{l}} & \frac{\partial^{2} Q\left(\boldsymbol{\theta}^{0}\right)}{\partial \lambda_{k} \partial \mu_{l}} \\
\frac{\partial^{2} Q\left(\boldsymbol{\theta}^{0}\right)}{\partial \mu_{k} \partial \mu_{s l}} & \frac{\partial^{2} Q\left(\boldsymbol{\theta}^{0}\right)}{\partial \mu_{k} \partial \phi_{l}} & \frac{\partial^{2} Q\left(\boldsymbol{\theta}^{0}\right)}{\partial \mu_{k} \partial \lambda_{l}} & \frac{\partial^{2} Q\left(\boldsymbol{\theta}^{0}\right)}{\partial \mu_{k} \partial \mu_{l}}
\end{array}\right]
\end{gathered}
$$

(C1) The expression of the elements of $Q_{k k}^{\prime \prime}\left(\boldsymbol{\theta}^{0}\right)$, for $k=1,2, \ldots, p$.

$$
\begin{aligned}
& \frac{\partial^{2} Q\left(\boldsymbol{\theta}^{0}\right)}{\partial \mu_{s k}^{2}}=2 M N \\
& \frac{\partial^{2} Q\left(\boldsymbol{\theta}^{0}\right)}{\partial \phi_{k}^{2}}=2 \sum_{m=1}^{M} \sum_{n=1}^{N} \mu_{s k}^{0}\left[x_{r}(m, n) \cos \left(w_{m n}\left(\boldsymbol{\theta}_{k}^{0}\right)\right)+x_{c}(m, n) \sin \left(w_{m n}\left(\boldsymbol{\theta}_{k}^{0}\right)\right)\right]+2 M N\left(\mu_{s k}^{0}\right)^{2} \\
& \frac{\partial^{2} Q\left(\boldsymbol{\theta}^{0}\right)}{\partial \lambda_{k}^{2}}=2 \sum_{m=1}^{M} \sum_{n=1}^{N} m^{2} \mu_{s k}^{0}\left[x_{r}(m, n) \cos \left(w_{m n}\left(\boldsymbol{\theta}_{k}^{0}\right)\right)+x_{c}(m, n) \sin \left(w_{m n}\left(\boldsymbol{\theta}_{k}^{0}\right)\right)\right]+2 N\left(\mu_{s k}^{0}\right)^{2} \sum_{m=1}^{M} m^{2} \\
& \frac{\partial^{2} Q\left(\boldsymbol{\theta}^{0}\right)}{\partial \mu_{k}^{2}}=2 \sum_{m=1}^{M} \sum_{n=1}^{N} n^{2} \mu_{s k}^{0}\left[x_{r}(m, n) \cos \left(w_{m n}\left(\boldsymbol{\theta}_{k}^{0}\right)\right)+x_{c}(m, n) \sin \left(w_{m n}\left(\boldsymbol{\theta}_{k}^{0}\right)\right)\right]+2 M\left(\mu_{s k}^{0}\right)^{2} \sum_{n=1}^{N} n^{2} \\
& \frac{\partial^{2} Q\left(\boldsymbol{\theta}^{0}\right)}{\partial \mu_{s k} \partial \phi_{k}}=2 \sum_{m=1}^{M} \sum_{n=1}^{N}\left[x_{r}(m, n) \sin \left(w_{m n}\left(\boldsymbol{\theta}_{k}^{0}\right)\right)-x_{c}(m, n) \cos \left(w_{m n}\left(\boldsymbol{\theta}_{k}^{0}\right)\right)\right] \\
& \frac{\partial^{2} Q\left(\boldsymbol{\theta}^{0}\right)}{\partial \mu_{s k} \partial \lambda_{k}}=2 \sum_{m=1}^{M} \sum_{n=1}^{N} m\left[x_{r}(m, n) \sin \left(w_{m n}\left(\boldsymbol{\theta}_{k}^{0}\right)\right)-x_{c}(m, n) \cos \left(w_{m n}\left(\boldsymbol{\theta}_{k}^{0}\right)\right)\right] \\
& \frac{\partial^{2} Q\left(\boldsymbol{\theta}^{0}\right)}{\partial \mu_{s k} \partial \mu_{k}}=2 \sum_{m=1}^{M} \sum_{n=1}^{N} n\left[x_{r}(m, n) \sin \left(w_{m n}\left(\boldsymbol{\theta}_{k}^{0}\right)\right)-x_{c}(m, n) \cos \left(w_{m n}\left(\boldsymbol{\theta}_{k}^{0}\right)\right)\right] \\
& \frac{\partial^{2} Q\left(\boldsymbol{\theta}^{0}\right)}{\partial \phi_{k} \partial \lambda_{k}}=2 \sum_{m=1}^{M} \sum_{n=1}^{N} m \mu_{s k}^{0}\left[x_{r}(m, n) \cos \left(w_{m n}\left(\boldsymbol{\theta}_{k}^{0}\right)\right)+x_{c}(m, n) \sin \left(w_{m n}\left(\boldsymbol{\theta}_{k}^{0}\right)\right)\right]+2 N\left(\mu_{s k}^{0}\right)^{2} \sum_{m=1}^{M} m \\
& \frac{\partial^{2} Q\left(\boldsymbol{\theta}^{0}\right)}{\partial \phi_{k} \partial \mu_{k}}=2 \sum_{m=1}^{M} \sum_{n=1}^{N} n \mu_{s k}^{0}\left[x_{r}(m, n) \cos \left(w_{m n}\left(\boldsymbol{\theta}_{k}^{0}\right)\right)+x_{c}(m, n) \sin \left(w_{m n}\left(\boldsymbol{\theta}_{k}^{0}\right)\right)\right]+2 M\left(\mu_{s k}^{0}\right)^{2} \sum_{n=1}^{N} n \\
& \frac{\partial^{2} Q\left(\boldsymbol{\theta}^{0}\right)}{\partial \lambda_{k} \partial \mu_{k}}=2 \sum_{m=1}^{M} \sum_{n=1}^{N} m n \mu_{s k}^{0}\left[x_{r}(m, n) \cos \left(w_{m n}\left(\boldsymbol{\theta}_{k}^{0}\right)\right)+x_{c}(m, n) \sin \left(w_{m n}\left(\boldsymbol{\theta}_{k}^{0}\right)\right)\right]+2\left(\mu_{s k}^{0}\right)^{2} \sum_{m=1}^{M} \sum_{n=1}^{N} m n
\end{aligned}
$$


(C2) The expressions of the elements of $Q_{k l}^{\prime \prime}\left(\boldsymbol{\theta}^{0}\right)$, for $k, l=1,2, \ldots, p$ and $k \neq l$.

$$
\begin{aligned}
& \frac{\partial^{2} Q\left(\boldsymbol{\theta}^{0}\right)}{\partial \mu_{s k} \partial \mu_{s l}}=2 \sum_{m=1}^{M} \sum_{n=1}^{N} \cos \left(w_{m n}\left(\boldsymbol{\theta}_{k}^{0}\right)-w_{m n}\left(\boldsymbol{\theta}_{l}^{0}\right)\right) \\
& \frac{\partial^{2} Q\left(\boldsymbol{\theta}^{0}\right)}{\partial \mu_{s k} \partial \phi_{l}}=-2 \mu_{s l}^{0} \sum_{m=1}^{M} \sum_{n=1}^{N} \sin \left(w_{m n}\left(\boldsymbol{\theta}_{k}^{0}\right)-w_{m n}\left(\boldsymbol{\theta}_{l}^{0}\right)\right) \\
& \frac{\partial^{2} Q\left(\boldsymbol{\theta}^{0}\right)}{\partial \mu_{s k} \partial \lambda_{l}}=-2 \mu_{s l}^{0} \sum_{m=1}^{M} \sum_{n=1}^{N} m \sin \left(w_{m n}\left(\boldsymbol{\theta}_{k}^{0}\right)-w_{m n}\left(\boldsymbol{\theta}_{l}^{0}\right)\right) \\
& \frac{\partial^{2} Q\left(\boldsymbol{\theta}^{0}\right)}{\partial \mu_{s k} \partial \mu_{l}}=-2 \mu_{s l}^{0} \sum_{m=1}^{M} \sum_{n=1}^{N} n \sin \left(w_{m n}\left(\boldsymbol{\theta}_{k}^{0}\right)-w_{m n}\left(\boldsymbol{\theta}_{l}^{0}\right)\right) \\
& \frac{\partial^{2} Q\left(\boldsymbol{\theta}^{0}\right)}{\partial \phi_{k} \partial \mu_{s l}}=2 \mu_{s k}^{0} \mu_{s l}^{0} \sum_{m=1}^{M} \sum_{n=1}^{N} \sin \left(w_{m n}\left(\boldsymbol{\theta}_{k}^{0}\right)-w_{m n}\left(\boldsymbol{\theta}_{l}^{0}\right)\right) \\
& \frac{\partial^{2} Q\left(\boldsymbol{\theta}^{0}\right)}{\partial \phi_{k} \partial \phi_{l}}=2 \mu_{s k}^{0} \mu_{s l}^{0} \sum_{m=1}^{M} \sum_{n=1}^{N} \cos \left(w_{m n}\left(\boldsymbol{\theta}_{k}^{0}\right)-w_{m n}\left(\boldsymbol{\theta}_{l}^{0}\right)\right) \\
& \frac{\partial^{2} Q\left(\boldsymbol{\theta}^{0}\right)}{\partial \phi_{k} \partial \lambda_{l}}=2 \mu_{s k}^{0} \mu_{s l}^{0} \sum_{m=1}^{M} \sum_{n=1}^{N} m \cos \left(w_{m n}\left(\boldsymbol{\theta}_{k}^{0}\right)-w_{m n}\left(\boldsymbol{\theta}_{l}^{0}\right)\right) \\
& \frac{\partial^{2} Q\left(\boldsymbol{\theta}^{0}\right)}{\partial \phi_{k} \partial \mu_{l}}=2 \mu_{s k}^{0} \mu_{s l}^{0} \sum_{m=1}^{M} \sum_{n=1}^{N} n \cos \left(w_{m n}\left(\boldsymbol{\theta}_{k}^{0}\right)-w_{m n}\left(\boldsymbol{\theta}_{l}^{0}\right)\right) \\
& \frac{\partial^{2} Q\left(\boldsymbol{\theta}^{0}\right)}{\partial \lambda_{k} \partial \mu_{s l}}=2 \mu_{s k}^{0} \sum_{m=1}^{M} \sum_{n=1}^{N} m \sin \left(w_{m n}\left(\boldsymbol{\theta}_{k}^{0}\right)-w_{m n}\left(\boldsymbol{\theta}_{l}^{0}\right)\right) \\
& \frac{\partial^{2} Q\left(\boldsymbol{\theta}^{0}\right)}{\partial \lambda_{k} \partial \phi_{l}}=2 \mu_{s k}^{0} \mu_{s l}^{0} \sum_{m=1}^{M} \sum_{n=1}^{N} m \cos \left(w_{m n}\left(\boldsymbol{\theta}_{k}^{0}\right)-w_{m n}\left(\boldsymbol{\theta}_{l}^{0}\right)\right) \\
& \frac{\partial^{2} Q\left(\boldsymbol{\theta}^{0}\right)}{\partial \lambda_{k} \partial \lambda_{l}}=2 \mu_{s k}^{0} \mu_{s l}^{0} \sum_{m=1}^{M} \sum_{n=1}^{N} m^{2} \cos \left(w_{m n}\left(\boldsymbol{\theta}_{k}^{0}\right)-w_{m n}\left(\boldsymbol{\theta}_{l}^{0}\right)\right) \\
& \frac{\partial^{2} Q\left(\boldsymbol{\theta}^{0}\right)}{\partial \lambda_{k} \partial \mu_{l}}=2 \mu_{s k}^{0} \mu_{s l}^{0} \sum_{m=1}^{M} \sum_{n=1}^{N} m n \cos \left(w_{m n}\left(\boldsymbol{\theta}_{k}^{0}\right)-w_{m n}\left(\boldsymbol{\theta}_{l}^{0}\right)\right) \\
& \frac{\partial^{2} Q\left(\boldsymbol{\theta}^{0}\right)}{\partial \mu_{k} \partial \mu_{s l}}=2 \mu_{s k}^{0} \sum_{m=1}^{M} \sum_{n=1}^{N} n \sin \left(w_{m n}\left(\boldsymbol{\theta}_{k}^{0}\right)-w_{m n}\left(\boldsymbol{\theta}_{l}^{0}\right)\right) \\
& \frac{\partial^{2} Q\left(\boldsymbol{\theta}^{0}\right)}{\partial \mu_{k} \partial \phi_{l}}=2 \mu_{s k}^{0} \mu_{s l}^{0} \sum_{m=1}^{M} \sum_{n=1}^{N} n \cos \left(w_{m n}\left(\boldsymbol{\theta}_{k}^{0}\right)-w_{m n}\left(\boldsymbol{\theta}_{l}^{0}\right)\right) \\
& \frac{\partial^{2} Q\left(\boldsymbol{\theta}^{0}\right)}{\partial \mu_{k} \partial \lambda_{l}}=2 \mu_{s k}^{0} \mu_{s l}^{0} \sum_{m=1}^{M} \sum_{n=1}^{N} m n \cos \left(w_{m n}\left(\boldsymbol{\theta}_{k}^{0}\right)-w_{m n}\left(\boldsymbol{\theta}_{l}^{0}\right)\right) \\
& \frac{\partial^{2} Q\left(\boldsymbol{\theta}^{0}\right)}{\partial \mu_{k} \partial \mu_{l}}=2 \mu_{s k}^{0} \mu_{s l}^{0} \sum_{m=1}^{M} \sum_{n=1}^{N} n^{2} \cos \left(w_{m n}\left(\boldsymbol{\theta}_{k}^{0}\right)-w_{m n}\left(\boldsymbol{\theta}_{l}^{0}\right)\right) \text {. }
\end{aligned}
$$


(D) The expression of the remainder $R_{M N}\left(\hat{\boldsymbol{\theta}}_{k}, \boldsymbol{\theta}^{0}\right)$ in the proof of Theorem 4.

$$
\begin{aligned}
R_{M N}\left(\hat{\boldsymbol{\theta}}_{k}, \boldsymbol{\theta}^{0}\right)= & \frac{1}{M N} \sum_{m=1}^{M} \sum_{n=1}^{N} s_{k}^{2}(m, n)\left[\cos \left(2 w_{m n}\left(\boldsymbol{\theta}_{k}^{0}\right)-2 w_{m n}\left(\hat{\boldsymbol{\theta}}_{k}\right)\right)-1\right] \\
& +\frac{1}{M N} \sum_{m=1}^{M} \sum_{n=1}^{N} \sum_{j \neq k} s_{j}^{2}(m, n) \cos \left(2 w_{m n}\left(\boldsymbol{\theta}_{j}^{0}\right)-2 w_{m n}\left(\hat{\boldsymbol{\theta}}_{k}\right)\right) \\
& +\frac{2}{M N} \sum_{m=1}^{M} \sum_{n=1}^{N} \sum_{j=1}^{p} e_{r}(m, n) s_{j}(m, n) \cos \left(w_{m n}\left(\boldsymbol{\theta}_{j}^{0}\right)-2 w_{m n}\left(\hat{\boldsymbol{\theta}}_{k}\right)\right) \\
& +\frac{2}{M N} \sum_{m=1}^{M} \sum_{n=1}^{N} \sum_{j=1}^{p} e_{c}(m, n) s_{j}(m, n) \sin \left(2 w_{m n}\left(\hat{\boldsymbol{\theta}}_{k}\right)-w_{m n}\left(\boldsymbol{\theta}_{j}^{0}\right)\right) \\
& +\frac{1}{M N} \sum_{m=1}^{M} \sum_{n=1}^{N} \sum_{i \neq j} s_{i}(m, n) s_{j}(m, n) \cos \left(w_{m n}\left(\boldsymbol{\theta}_{i}^{0}\right)+w_{m n}\left(\boldsymbol{\theta}_{j}^{0}\right)-2 w_{m n}\left(\hat{\boldsymbol{\theta}}_{k}\right)\right) \\
& +\frac{1}{M N} \sum_{m=1}^{M} \sum_{n=1}^{N}\left[e_{r}^{2}(m, n)-e_{c}^{2}(m, n)\right] \cos \left(2 w_{m n}\left(\hat{\boldsymbol{\theta}}_{k}\right)\right) \\
& +\frac{2}{M N} \sum_{m=1}^{M} \sum_{n=1}^{N} e_{r}(m, n) e_{c}(m, n) \sin \left(-2 w_{m n}\left(\hat{\boldsymbol{\theta}}_{k}\right)\right)
\end{aligned}
$$

(E) The proof of " $f_{M N}\left(\hat{\boldsymbol{\theta}}, \boldsymbol{\theta}^{0}\right) \rightarrow 0$ a.s." in the proof of Theorem 5 .

$$
\begin{aligned}
& \frac{1}{M N} \sum_{m=1}^{M} \sum_{n=1}^{N}\left\{\sum_{k=1}^{p}\left[\mu_{s k}^{0} \cos \left(w_{m n}\left(\boldsymbol{\theta}_{k}^{0}\right)\right)-\hat{\mu}_{s k} \cos \left(w_{m n}\left(\hat{\boldsymbol{\theta}}_{k}\right)\right)\right]\right\}^{2} \\
\leq & \frac{p}{M N} \sum_{k=1}^{p} \sum_{m=1}^{M} \sum_{n=1}^{N}\left[\mu_{s k}^{0} \cos \left(w_{m n}\left(\boldsymbol{\theta}_{k}^{0}\right)\right)-\hat{\mu}_{s k} \cos \left(w_{m n}\left(\hat{\boldsymbol{\theta}}_{k}\right)\right)\right]^{2} \\
\leq & \frac{2 p}{M N} \sum_{k=1}^{p} \sum_{m=1}^{M} \sum_{n=1}^{N}\left\{\left(\mu_{s k}^{0}-\hat{\mu}_{s k}\right)^{2} \cos ^{2}\left(w_{m n}\left(\boldsymbol{\theta}_{k}^{0}\right)\right)+\hat{\mu}_{s k}^{2}\left[\cos \left(w_{m n}\left(\boldsymbol{\theta}_{k}^{0}\right)\right)-\cos \left(w_{m n}\left(\hat{\boldsymbol{\theta}}_{k}\right)\right)\right]^{2}\right\} \\
\leq & 2 p \sum_{k=1}^{p}\left(\mu_{s k}^{0}-\hat{\mu}_{s k}\right)^{2}+\frac{2 p}{M N} \sum_{k=1}^{p} \sum_{m=1}^{M} \sum_{n=1}^{N} \hat{\mu}_{s k}^{2}\left[w_{m n}\left(\boldsymbol{\theta}_{k}^{0}\right)-w_{m n}\left(\hat{\boldsymbol{\theta}}_{k}\right)\right]^{2} \\
\rightarrow & 0 \text { a.s. }
\end{aligned}
$$

where the first inequality follows from Cauchy-Schwarz inequality, the second inequality follows from the fact $2 x^{2}+2 y^{2}-(x+y)^{2} \geq 0$, the third inequality follows from Taylor series expansion and the last step follows from the results in Theorems 1 and 2. Along the same line, it also can be shown that

$$
\frac{1}{M N} \sum_{m=1}^{M} \sum_{n=1}^{N}\left\{\sum_{k=1}^{p}\left[\mu_{s k}^{0} \sin \left(w_{m n}\left(\boldsymbol{\theta}_{k}^{0}\right)\right)-\hat{\mu}_{s k} \sin \left(w_{m n}\left(\hat{\boldsymbol{\theta}}_{k}\right)\right)\right]\right\}^{2} \rightarrow 0 \text { a.s. }
$$

Therefore, it can be obtained from (A.4) in the proof of Theorem 1 that $f_{M N}\left(\hat{\boldsymbol{\theta}}, \boldsymbol{\theta}^{0}\right) \rightarrow 0$ a.s. 